\title{
Three-Dimensional Finite Element Analysis on En-Mass Retraction of Maxillary Anterior Teeth With the Clear Aligner Technique
}

\section{Xiaosong Xiang}

Department of Orthodontics, Afliliated Stomatology Hospital of Guangzhou Medical University, Guangzhou Key Laboratory of Basic and Applied Research of Oral Regenerative Medicine.

\section{Yujing Bai}

Department of orthodontics, Tianjin Stomatological Hospital, School of Medicine, Nankai University

\section{Xiaodong Wei}

Department of orthodontics, Tianjin Stomatological Hospital, School of Medicine, Nankai University

\section{Yuebai Ji}

Department of orthodontics, Tianjin Stomatological Hospital, School of Medicine, Nankai University

\section{Yunyun Yu}

Department of orthodontics, Tianjin Stomatological Hospital, School of Medicine, Nankai University

\section{Song Cang ( $\nabla$ yiyijoy1@yeah.net)}

Department of orthodontics, Tianjin Stomatological Hospital, School of Medicine, Nankai University

\section{Research Article}

Keywords: Finite element analysis, Orthodontic retraction of maxillary anterior teeth, Biomechanics, CA

Posted Date: March 15th, 2021

DOI: https://doi.org/10.21203/rs.3.rs-288345/v1

License: (c) This work is licensed under a Creative Commons Attribution 4.0 International License. Read Full License 


\section{Abstract \\ Objective}

This study aimed to investigate the biomechanics effects during en囚mass retraction of maxillary anterior teeth with the clear aligner (CA) system by three®dimensional finite element method, which provides a theoretical basis for orthodontics and CA exploitation.

\section{Methods}

Cone-beam computer tomography (CBCT) was used to scan a normal occlusion adult volunteer who meeting the modeling standards from the Hospital of Stomatology Nankai University. A finite element model including maxillary teeth, periodontal ligament (PDL), alveolar bone and CA was established by Mimics 17.0, Geomagic Studio 12.0, and UG NX software packages. The analysis of material properties of the model set the contact, constraint, and load was performed using the ANSYS Workbench 19.0. CA was employed to simulate the $0.2 \mathrm{~mm}$ en『mass retraction of anterior teeth and analyze the results.

\section{Results}

The movement of anterior teeth with an inclination of the lingual and distal crown was observed to have the tendency overbite increasing and the posterior teeth tending to incline mesially. The maximum displacement of the central incisor, lateral incisor, canine, second premolar, and first molar were 0.320, 0.290, 0.287, 0.350, and 0.230 mm, respectively. The stress distribution of PDL and alveolar fossa were concentrated in the neck and apical areas of teeth. The stress distribution of PDL on both sides of the extraction space was broad which consistent with the trend of tooth movement. The entire CA had a tendency of dislocation $\square$ displacement and deformation. The CA showed stress concentration at the junction of adjacent teeth and the joints of the posterior teeth were the main stress concentration areas.

\section{Conclusion}

To achieve en囚mass retraction of maxillary anterior teeth with the CA system, we should pay attention to the orthodontic optimization design, the retraction procedure of torque, intrusion and anchorage protection of posterior teeth, and the CA retention.

\section{Introduction}

With progressively increased requirements for aesthetics and comfort during orthodontic treatment, which promotes the population of clear aligner (CA). However, initially clear aligner treatment (CAT) is only suitable for some simple and specific malocclusions such as closing scattered gaps,tipping, and correction of relapsing cases. Furthermore, the control of extraction cases is subject to numerous limitations (e.g., common cases of premolar extraction). For the extraction of premolars, the expected effect of the CA is still difficult to achieve [1,2]. However[premolar extraction is most commonly used in orthodontic treatment, especially in Asian populations [3]. 
As revealed from clinical studies, during CAT of premolar extraction, the loss of anterior torque, deepening of the overlay, and mesial tipping of the molars tend to be consistent with the "roller coaster" effect in conventional orthodontic treatment $[1,4,5]$. Firstly, it is difficult for clear aligners to gain reasonable result in the extraction cases, mainly due to that the biomechanical mechanism of the CA technique is unclear [6, 7]. Previous research had shown that CA can generate force and motion, and attachments can enhance the control of tooth movement. However, there is no clear theoretical support for how to generate motion and how attachments can assist CA to move teeth as expected [8]. Specific to conventional orthodontic treatment, force and moment are exploited in the teeth via the interaction between the arch wires and brackets, as an attempt to move the tooth as expected. In contrast to the above-mentioned studies, after the CA is placed according to the simulated target position, complicated forces are applied to the teeth through elastic deformation and then realized the teeth movement. [6, 9]. As a matter of fact, it is really difficult to understand what kinds of force realizes tooth movement of digital simulation. Secondly, during the orthodontic treatment, auxiliary devices (e.g., attachment, elastics, micro screw) are also required. [6, 10]. It is unlikely to clarify the respective roles of aligners and auxiliary devices in the system [11-15]. Besides, there has been no material diaphragm capable of simultaneously achieving strong retention, good elasticity and slow force attenuation of the CA. Fortunately, in the last few years, with the leaping forward of CA technology and in-depth research on biomechanical mechanisms, the performance of the diaphragm material has been improved, which has enabled the CA to be gradually used in the orthodontic treatment of complex extraction cases $[16,17]$. In clinical, a series of small tooth movements are completed by wearing a range of aligners mentioned above, and finally, the teeth are positioned $[10,18]$.

Therefore, to reveal the system biomechanical principles, the biomechanical principles of CA without assistive devices to close the extraction space of the premolars should be initially studied, and the clinical and later series of studies should be underpinned theoretically. Considering the complexity of orthodontic biomechanics and the principles of medical ethics, biomechanics in related fields cannot be effectively studied in vivo [10]. Therefore, the use of the finite element method in vitro simulation research makes up for a defect of the current research,and provides a better research direction for orthodontic biomechanics research. Considerable studies reported that the three-dimensional finite element method is a harmless, effective simulation, reliable results, in line with clinical practice, and suitable for fine structure principles and biomechanics in vitro research methods. Numerous scholars employed this method to analyze the tooth movement, centre of resistance, stress and strain of periodontal ligament, alveolar bone and appliance. Fortunately, they have achieved reliable results to a certain extent [19-21]. The study aimed to investigate the biomechanical principle of the maxillary anterior teeth retraction in the extraction of the first premolars with CA by employing the three-dimensional finite element method. This study will theoretically provide a basis for a subsequent series of studies and guide the clinical application of CA.

\section{Materials And Methods}

\subsection{Model construction}

Jaws and dentition of the volunteer were scanned by CBCT (EWOO-VATECH, South Korea), and Digital Imaging and Communications in Medicine(DICOM)format data were imported into Mimics 17.0 (Materialise, Belgium). Data of alveolar bone, teeth and other cementum were extracted by thresholding segmentation of $900 \mathrm{HU}$ to $3095 \mathrm{HU}$. After gray value selection, region growing, noise reduction and binarization processing, the three-dimensional model (3D model) of the maxillary dentition and jaw were imported into Geomagic Studio 12.0 (Raindrop, USA) for repairing, smoothing and glossing to generate a high-precision 3D solid model. To solidify the geometric model, the 3D model was imported into UG NX 12.0 (UGS, USA). To visualize the extraction of the maxillary first premolar, the bilateral 
maxillary first premolars were deleted to simulate, and according to the scientific literature, the root of the teeth was spread uniformly along the outer surface by $0.25 \mathrm{~mm}$ continuously to obtain the PDL model [10, 22]. The maxillary anterior teeth were retracted bodily by $0.2 \mathrm{~mm}$ in sagittal direction [23], and the dentition was counted as $\mathrm{M}$. Then $\mathrm{M}$ was imported into Geomagic Studio 12.0 software to process the space between every two teeth separately. Then the outer surface of the crown was removed as an inner surface of CA, sliced through the boundary curve of the aligner. The thickness of the template is $0.5 \mathrm{~mm}$ and the Boolean operation is carried out with $\mathrm{M}$ to obtain the solid geometric model of CA (Fig. 1) [10]. The solid models were combined into an assembly (Fig. 1). The geometric solid model was imported into Workbench 19.0 (ANSYS, USA) for parameter setting, mesh division, loading and other operation to simulate the bodily retraction of the maxillary anterior teeth by $0.2 \mathrm{~mm}$ in the sagittal direction. And postprocessing function of the software was used to evaluate the data. All methods were carried out in accordance with the guidelines and regulations of the Institutional Animal Care Committee of Nankai University. All experiments procedures and methods were performed following the protocol approved by the Institutional Animal Care Committee of Nankai University. All experiments and methods had confirmed that informed consent was obtained from all subjects

\subsection{Material properties, interaction and boundary setting}

The substance was defined with a continuously homogeneous and isotropic; the orbital floor of the maxillary alveolar bone was a fixed constraint. There was frictional interaction between the crown and the CA, with a friction coefficient of 0.2 [23]. Binding restrictions were found between the teeth of the PDL and the alveolar bone. The elastic modulus of the alveolar bone, dental body, PDL and CA was 2,000, 20,000, 0.05 and 450 Mpa respectively. Poisson's ratio was $0.30,0.30,0.35$ and 0.40 respectively [24].

\subsection{Mesh division and results}

CA used triangular six-node shell elements and other models adopt tetrahedral ten-node elements, both of which have properties such as plasticity, wiggling, large deformation, and high tensility. Results of the division: the number of nodes for the whole model, alveolar bone, teeth, PDL, and CA is 1138046, 257810, 569455, 117193, and 193598; the number of components is $726808,162931,390852,58026$, and 114999

\subsection{Coordinate system setting}

The system of global cooperation had been implemented. The parallel occlusal plane pointing to the left side of the patient was the positive orientation of the $\mathrm{X}$-axis and the reverse direction was negative. The direction perpendicular to the $X$-axis pointing to the tooth apex was the positive direction of the Z-axis, and the reverse direction was negative. The direction of the incisor perpendicular to the $\mathrm{X}$ and Z-axis is the positive direction of the $\mathrm{Y}$-axis, and the opposite direction is the negative direction.

\subsection{Calculation and analysis}

The model was bilaterally symmetrical and the right maxillary model was chosen for the study. Analysis items: original tooth displacement pattern and displacement value; periodontal tissue stress distribution; CA deformation trend and stress distribution.

\section{Results}

\subsection{Maxillary-dentition-PDL-CA three-dimensional finite element model}


In this study, the high-quality three-dimensional finite element models of maxillary teeth, periodontal ligament, alveolar bone, clear aligner and the assembly of the above models were obtained.

\subsection{Initial displacement trend and displacement value of teeth}

As Fig. 2-3 and Table 1 indicated that the measurement of the crown for the midpoint of the incisor tip, the canine cusp, the buccal cusp of the second premolar, and the buccal and lingual cusps of the first molar, as well as the apical measurement for tooth apex. The original displacement value and the displacement trend of the maxillary teeth are associated with the tipping movement of the crowns and roots in the opposite direction. The crown of the incisor tilted toward the distal lingual side and the tooth root of the incisor tipped toward the mesial labial side. The movement of the canine was similar to that of the incisor. The crowns of the central incisor, the lateral incisors, and the canine saw the distal inclination was steadily rising and the lingual inclination was gradually declining. They extrude perpendicularly to the occlusal orientation of the maxillary anterior teeth. The crowns of the posterior teeth tilted to the palatal mesial side and the roots tilted to the distal side of the jaw. The crown of the second premolar intruded vertically upwards, and the crown of the first molar had the mesial marginal edge intruded and the distal marginal edge extruded.

Table 1

Initial displacement of maxillary anterior teeth on $\mathrm{X}, \mathrm{Y}$ and $\mathrm{Z}$ axes $(\mathrm{mm})$

\begin{tabular}{|c|c|c|c|c|}
\hline Tooth & Position & $\mathrm{X}$-axis & Y-axis & Z-axis \\
\hline \multirow[t]{2}{*}{ Central incisor } & crown & -0.02597 & -0.13336 & -0.23133 \\
\hline & root tip & 0.00724 & 0.04616 & -0.08820 \\
\hline \multirow[t]{2}{*}{ Lateral incisor } & crown & -0.21951 & -0.22496 & -0.15497 \\
\hline & root tip & 0.08623 & 0.07647 & -0.05400 \\
\hline \multirow[t]{2}{*}{ Canine } & crown & -0.27002 & -0.03771 & -0.01360 \\
\hline & root tip & 0.03566 & 0.03715 & -0.02142 \\
\hline \multirow[t]{2}{*}{ Second premolar } & crown & 0.08141 & 0.30111 & 0.01008 \\
\hline & root tip & -0.03286 & -0.13297 & -0.00267 \\
\hline \multirow[t]{7}{*}{ First molar } & Mesial buccal cusp & 0.05133 & 0.03523 & 0.02731 \\
\hline & Mesial lingual cusp & 0.04913 & 0.02863 & 0.01610 \\
\hline & Distal buccal cusp & 0.04857 & 0.02082 & -0.02108 \\
\hline & Distal lingual cusp & 0.07070 & 0.02105 & -0.02410 \\
\hline & Mesial apex & -0.04498 & -0.02054 & 0.00088 \\
\hline & Distal apex & -0.04719 & -0.01927 & -0.00143 \\
\hline & Palatal apex & -0.02016 & -0.00962 & -0.00055 \\
\hline
\end{tabular}

\subsection{Stress distribution of periodontal tissue}


As shown in Fig. 3, PDL stress concentrated in the cervical and apical regions of the teeth or the lingual and distal surfaces of the anterior teeth. Stress amounts were also found in the labial side of the lateral incisor near the distal and mesial surfaces and the mesial surfaces of the canine. Stress in the apical area of the inner surface of the anterior teeth PDL was larger than that in other regions. Stress in the posterior tooth region is mostly in the cervical area adjacent to the distal margin, the apical area and inner surface of the teeth. The highest tension of the anterior teeth is all in the cervical region of the teeth. It was in the mesial cervical region for the second premolar. The first molar was in the distal buccal and palatal apical area and the inner surface of the corresponding PDL. The details are shown in Table 2. Figure 5 exhibited that tensile stress and compressive stress of PDL were defined as the negative/positive definition of the maximum main stress. Tensile stress-compressive stress distribution was consistent with the trend of tooth displacement. In the lingual region, the compressive stress of the incisor was near to the distal surface and apical portion of the labial side of the tooth neck, and tensile stress was present in the labial cervical area and the lingual apex. The canine was similar to the incisor except the tension was heavier. The compressive stress of posterior teeth was present in the mesial cervical and distal apex regions, and the tensile stress was present in the distal cervical and mesial apex area. According to Table 2, the absolute value of the peak tensile stress was greater than the compressive stress, and the stress on both sides of the tooth extraction region was high. Figure 4 indicated the tension of the alveolar socket localized in the cervical region of the tooth. It was larger in the anterior tooth area, small in the apical area, and not apparent in other areas.

Table 2

Comparison of peak PDL stress in maxillary teeth (MPa)

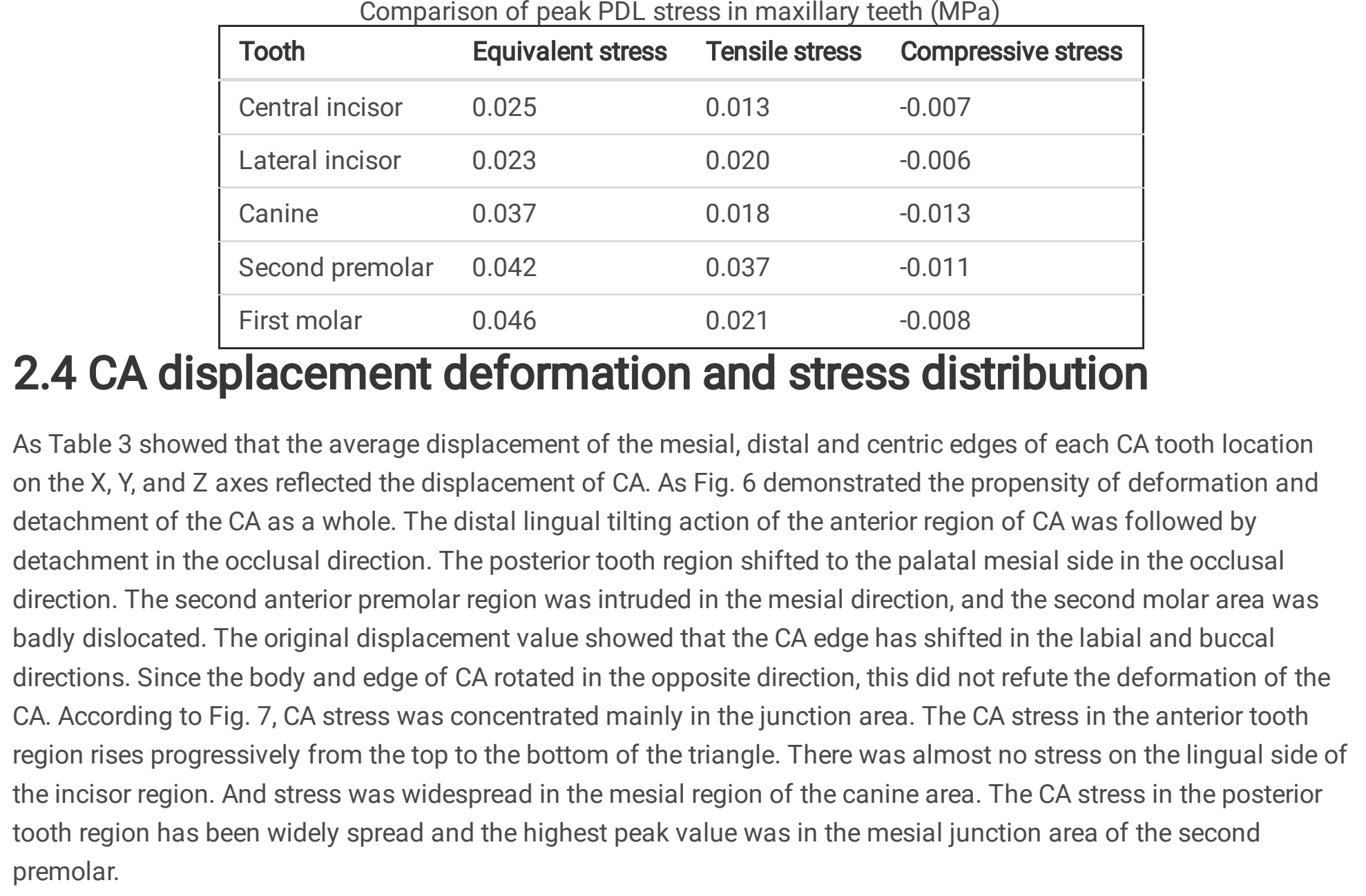


Table 3

Initial displacement of CA on $X, Y$ and $Z$ axes $(\mathrm{mm})$.

\begin{tabular}{|c|c|c|c|c|c|c|}
\hline \multirow[t]{2}{*}{ Position } & \multicolumn{2}{|l|}{$\mathrm{X}$-axis } & \multicolumn{2}{|l|}{ Y-axis } & \multicolumn{2}{|l|}{ Z-axis } \\
\hline & labial/buccal & lingual/palatal & labial/buccal & lingual/palatal & labial/buccal & lingual/palatal \\
\hline $\begin{array}{l}\text { Central } \\
\text { incisor }\end{array}$ & -0.014 & -0.016 & 0.128 & 0.174 & -0.047 & 0.061 \\
\hline $\begin{array}{l}\text { Lateral } \\
\text { incisor }\end{array}$ & -0.083 & -0.097 & 0.095 & 0.173 & -0.024 & 0.051 \\
\hline Canine & -0.053 & -0.125 & -0.167 & -0.177 & -0.025 & 0.037 \\
\hline $\begin{array}{l}\text { 1st } \\
\text { premolar }\end{array}$ & -0.009 & -0.003 & 0.074 & 0.118 & -0.019 & -0.052 \\
\hline $\begin{array}{l}\text { 2nd } \\
\text { premolar }\end{array}$ & -0.016 & -0.075 & 0.209 & 0.181 & -0.029 & -0.040 \\
\hline $\begin{array}{l}1 \mathrm{st} \\
\text { molar }\end{array}$ & -0.015 & -0.037 & 0.198 & 0.137 & -0.120 & -0.072 \\
\hline
\end{tabular}

\section{Discussion}

The finite element method was a numerical method for mechanical analysis. With structural discretization as the heart, it used a finite range of easy-to-analyze elements to express and address complex objects. The finite element approach was an efficient method of mechanical analysis with good numerical precision. It was specifically suited to stress-strain problems in engineering structure and solid mechanics, such as dental biomechanics [6]. The finite element approach was currently the only in vivo research method for clear aligner biomechanics that requires periodontal tissue for assessment. And the stress and displacement of any portion of the model loaded can be calculated [7]. This research, therefore, followed a three-dimensional finite element approach to investigate the biomechanical effects of CAT bodily retraction of maxillary anterior teeth.

\subsection{Research modeling and loading effectiveness}

The cornerstone of the architecture of a finite element model construction is the simplification of the geometric morphology of the research sample and the idealization of the mechanical properties of the components. The fact that the model can represent the mechanical properties of the simulated object or the degree to which a realistic simulation can be performed decides the usefulness of the finite element model. One of the methods used to verify validity was to equate the effects of the estimation of the developed finite element model with the actual clinical situation. If the outcomes of the simulation estimation are consistent with explicit clinical mechanical effects, the validity of the finite element model would be justified. In this analysis, a series of solid models of maxillary dentition, PDL, alveolar bone and CA were built based on a simulation of maxillary first premolars extraction. The findings of the study revealed that the maxillary anterior teeth had lingual and distal tipping movements accompanied by occlusal extrusion, and the maxillary posterior teeth exhibited mesial tipping movement accompanied by an intrusion trend during the maxillary anterior teeth retraction process. The research results were consistent with clinical experience and close to those of other researchers $[9,23,24]$ which demonstrates the rationality of this research.

\subsection{Application of CAT system in orthodontics practice}




\subsubsection{The impact of CAT on the maxillary dentition}

According to studies, CAT extracting premolars had weak three-dimensional control over teeth, particularly in the sagittal direction which can trigger problems perpendicularly. In most cases, fixed equipment would be used to address the tooth inclination problem on both sides of the extraction area at the later stage of therapy [5]. The actual results of this study were inconsistent with the original intention of the design. All of the anterior teeth exhibited lingual crown and labial root tipping motions, similar to those of other scholars, suggesting that the pure CA that wraps the teeth in clinical applications cannot produce the optimal force that passes through the dental resistance centre [25]. It was also important to pay attention in clinical practice to the movement of the teeth and the thickness of the alveolar bone wall on the labial, buccal and lingual sides of the teeth, to avoid lingual tipping, deepening of the overbite, and even fenestration and dehiscence of the teeth [26]. Firstly, the optimization of the corrective technique for torque control, interference and retraction and installation of auxiliary equipment (such as attaching torque control attachment to the canine to produce a reversing moment to combat tipping motions and designing Power Ridge on anterior torque control teeth) should be used to improve the torque control impact clinically [9, 27]. Studies had shown that Power Ridge can maintain strong torque control for anterior teeth [28]. Power Ridge will almost escape torque loss for torque regulation within $10^{\circ}$. For torque control beyond $10^{\circ}$, the addition of torque attachments and Power Ridge on the tooth surface each had about 50\% torque intake [11]. Second, the attachment should be used to improve the retention of CA to maximize the representation of torque force and to retain generalized space in the anterior teeth during the retraction process, thereby improving the CA wrapping of the teeth and better regulating the dental axis [29]. Thirdly, preset over-correction for maxillary anterior teeth can be applied to the clinical check software design to prevent unnecessary torque loss and then palatal tipping [30]. According to the research, the posterior teeth have mesial inclination during the retraction process of the anterior teeth. It was also necessary to design a strategy to improve the anchorage to avoid loss of resistance, such as a two-step retraction, a frog pattern retraction, an optimized anchorage attachment in the posterior teeth built concerning the Tweed-Merrifield classic square arch wire orthodontic anchorage planning procedure to minimize the anterior inclination of the posterior teeth or an implantation anchorage [1, 31]. For apparent mesial tipping, a combination of segmental arch wire correction can be considered or restarted the treatment [32]. The perpendicular anterior teeth were extruded and the posterior teeth intruded, indicating that the perpendicular control needs to be strengthened in a direct aligner treatment. To improve the efficiency of CA treatment for premolar extraction cases, it was necessary to enhance the controlling performance over three-dimensional tooth movement, especially in the sagittal direction.

\subsubsection{Biomechanical analysis of CAT system}

The tooth was bound by PDL to the neighbouring alveolar bone, forming a structural unit of typical morphology and function. After the orthodontic force was applied to the tooth, the proper force was moved to the periodontal tissue, resulting in periodontal tissue repair and tooth displacement [33]. CA applied orthodontic force through elastic rebound after deformation. This force functions in a complicated way and was critical for the biomechanical analysis of the CAT system [12]. Some scholars analyzed the bodily distal movement of the canine and anterior teeth by three-dimensional finite factor and noticed that the PDL compressive stress in the anterior teeth was distributed in the distal cervical and mesial apical areas, and tensile stress was distributed in the mesial cervical and distal apical areas, similar to the findings of this research $[9,24]$. Stress was also concentrated in the corresponding regions of the alveolar bone. It was speculated that there would be a great deal of stress in the related region of the apical. Clinical care should also be taken against the possibility of periodontal injuries, such as hyaline degeneration and also the risk of root resorption. Currently, some reports were already available for root resorption triggered by CAT [33]. CA stress was concentrated more in the junction area of adjacent spaces, in particular the extraction area, indicating that 
this area was prone to damage and that the materials are vulnerable to breakage. The structural configuration of the aligner in the extraction regions should be improved to prevent damage to its mechanical system due to CA breakdown of the CA and the adjustment in the effective propagation of force, which would compromise the efficiency of the appliance. During the retraction process of the anterior teeth, the stress in the incisor region of CA was primarily spread on the labial side and there was almost no stress on the lingual side. Canine stress was more in the mesial area than in the distal area, indicating that CA generates pressure in retraction of incisors and canine distal movement as an orthodontic force. This suggested that CA was a pressure appliance, rather than an appliance that generates pulling force.

\subsection{Material development and design for CAT system}

CA scientifically adopted diaphragms of different thicknesses and the thickness of the diaphragm affect the value of single-step tooth movement and the entire orthodontic procedure. Diaphragms with differing thicknesses have distinct properties. The $1.0 \mathrm{~mm}$ diaphragm had wide elastic modulus and high retaining stress, suitable for the maintenance of dentition. The $0.5 \mathrm{~mm}$ diaphragm was the smallest one. And the $0.75 \mathrm{~mm}$ diaphragm was medium, with strong plasticity and a small elastic modulus, favourable for tooth movement and convenient to wear, and a broad tooth movement can be designed [12]. Therefore, it was impossible to achieve ideal tooth movement by CA alone in clinical practice. The benefits of CA were simple alignment and closure of narrow spaces. However, there were also some defects in extraction situations, such as teeth on both sides of extraction space tipping into the extraction space, difficult control over the torque of anterior teeth, and overbite deepening $[1,12,27]$. Therefore, in the clinical practice of the CA system, in addition to the implementation of attachments and other auxiliary equipment for target dental movement, the presumption was to develop a biomechanical-oriented CA based on a thorough knowledge of CAT biomechanics and to design customized CA using CAD/CAM technologies. such as increasing CA strength at the site of significant deformation to avoid a variety of problems caused by deformation. And CA with different physical properties can also be designed according to different orthodontic goals in different orthodontic stages and for different tooth positions [34]. In combination with auxiliary devices, a biomechanically oriented CA designed in such a way that meets the target tooth movement can achieve higher tooth movement efficiency. In the case of CA detachment, retention was mainly enhanced through retention attachments and other devices. On one hand, the design and development of attachments can be strengthened. On the other hand, the material structure of the diaphragm can be modified assumedly to increase the retention by forming natural chemical bonds in saliva between the diaphragm and the teeth. A study in the ferroelectrics letters section suggested that the plastic that had been used to fabricate Essex aligners had in fact a piezoelectric property and be used as ultrasound transducers [35]. This property was important in orthodontic treatment for one of the fundamental theories of the biology of orthodontic tooth movement was the piezoelectric theory [35]. According to the researches, aligners might produce a flow of electric current that could help in either tooth movement and /or bone remodeling when the patient bites on the aligners. It had been reported that ultrasound can minimize orthodontically induced tooth root resorption and enhances dental tissue formation [36]. In the future area of research and development, clear aligners could enhance tooth movement, preventing teeth root resorption, and also enhancing bone remodeling.

\section{Conclusion}

1. In this study, the CBCTs of the volunteers were scanned to acquire data in DICOM format, and Mimics software was employed for 3D reconstruction. Geomagic Studio reverse engineering and UG NX and Workbench software was used to successfully obtain three-dimensional finite element models of maxillary alveolar bone, teeth, periodontal ligament, and non-bracket-supported clear aligners. The model has high accuracy, geometric similarity and mechanical similarity can be used in simulation research. As demonstrated by the displacement results of this study, 
the anterior crown of the tooth and root moved obliquely in the opposite direction during adduction of the maxillary anterior teeth. In this study, the posterior teeth were not designed to move, whereas the result showed that the back teeth had achieved media-oblique movement. It was therefore revealed that the orthodontic force of the tooth cannot pass through the impedance centre of the tooth. The vertical front teeth seem to be elongated, and the back teeth tend to be depressed. The periodontal tissue had been unevenly stressed. The stress of the periodontal ligament was concentrated mainly in the neck and apical areas of the tooth. There was a concentration of stress on the inner surface of the alveolar bone and the corresponding area of the periodontal ligament. The whole clear aligner was deformed, and there was a tendency to slip simultaneously in the upper incisor and molar areas, especially in the second molar area. The clear aligner had stress concentration at the adjacent points between the teeth, especially in the extraction area, which demonstrated that this area of the appliance was easily damaged.

6. The distribution of stress in the anterior teeth of the appliance was significantly different. Stress occurs on the inner surface of the labial side in the incisor area, while stressing distribution on the lingual side almost nonexistent. There were more stress zones in the center of the canine than in the distal region. The results indicated that the strength of the invisible braces was moved as the incisors come in and the fangs move in the middle, suggesting that the invisible braces are more of a thrust rather than tension.

\section{Declarations}

\section{Acknowledgements}

The authors acknowledge the members in Tianjin Key Laboratory of Oral and Maxillofacial Function Reconstruction for their invaluable cooperation in preparing this study and the statistical analysis.

\section{Conflict of interest}

None to declare.

\section{References}

1. Dai, F. F., Xu, T. M. \& Shu, G. Comparison of achieved and predicted tooth movement of maxillary first molars and central incisors: First premolar extraction treatment with Invisalign. Angle Orthod. 8, 679-687 (2019).

2. Zhu, Y., Li, X. \& Lai, W. Treatment of Severe Anterior Crowding with the Invisalign G6 First-Premolar Extraction Solution. J Clin Orthod. 53, 459-469 (2019).

3. RK LKJ. Treating Bimaxillary Protrusion and Crowding with the Invisalign G6 First Premolar Extraction Solution and Invisalign Aligners. APOS Trends Orthod. 8, 219-224 (2018).

4. Venugopal, A., Manzano, P. \& Rengalakshmi, S. (2020) A Novel Temporary Anchorage Device Aided Sectional Mechanics for Simultaneous Orthodontic Retraction and Intrusion. Case Rep Dent, 2020:5213936.

5. Papadimitriou, A., Mousoulea, S., Gkantidis, N. \& Kloukos, D. Clinical effectiveness of Invisalign® orthodontic treatment: a systematic review. Prog Orthod. 19, 37 (2018).

6. Barone, S., Paoli, A., Razionale, A. V. \& Savignano, R. Computational design and engineering of polymeric orthodontic aligners. Int J Numer Method Biomed Eng. 33, (2017). e2839

7. White, D. W., Julien, K. C., Jacob, H., Campbell, P. M. \& Buschang, P. H. Discomfort associated with Invisalign and traditional brackets: A randomized, prospective trial. Angle Orthod. 87, 801-808 (2017). 
8. Yokoi, Y. et al. (2019) Effects of Attachment of Plastic Aligner in Closing of Diastema of Maxillary Dentition by Finite Element Method.J Healthc Eng,1075097.

9. Gomez, J. P., Pena, F. M., Martinez, V., Giraldo, D. C. \& Cardona, C. I. Initial force systems during bodily tooth movement with plastic aligners and composite attachments: A three-dimensional finite element analysis. Angle Orthod. 85, 454-460 (2015).

10. Cortona, A., Rossini, G., Parrini, S., Deregibus, A. \& Castroflorio, T. Clear aligner orthodontic therapy of rotated mandibular round-shaped teeth: A finite element study. Angle Orthod. 90, 247-254 (2020).

11. Simon, M., Keilig, L., Schwarze, J., Jung, B. A. \& Bourauel, C. Forces and moments generated by removable thermoplastic aligners: incisor torque, premolar derotation, and molar distalization. Am J Orthod Dentofacial Orthop. 145, 728-736 (2014).

12. Iliadi, A., Koletsi, D. \& Eliades, T. Forces and moments generated by aligner-type appliances for orthodontic tooth movement: A systematic review and meta-analysis. Orthod Craniofac Res. 22, 248-258 (2019).

13. Gao, L. \& Wichelhaus, A. Forces and moments delivered by the PET-G aligner to a maxillary central incisor for palatal tipping and intrusion. Angle Orthod. 87, 534-541 (2017).

14. Elkholy, F., Schmidt, F., Jäger, R. \& Lapatki, B. G. Forces and moments delivered by novel, thinner PET-G aligners during labiopalatal bodily movement of a maxillary central incisor: An in vitro study. Angle Orthod. 86, 883-890 (2016).

15. Elkholy, F., Panchaphongsaphak, T., Kilic, F., Schmidt, F. \& Lapatki, B. G. Forces and moments delivered by PET-G aligners to an upper central incisor for labial and palatal translation. J Orofac Orthop. 76, 460-475 (2015).

16. Rossini, G., Parrini, S., Castroflorio, T., Deregibus, A. \& Debernardi, C. L. Efficacy of clear aligners in controlling orthodontic tooth movement: a systematic review. Angle Orthod. 85, 881-889 (2015).

17. Robertson, L. et al. Effectiveness of clear aligner therapy for orthodontic treatment: A systematic review. Orthod Craniofac Res. 23, 133-142 (2020).

18. Knop, L., Gandini, L. G. Jr., Shintcovsk, R. L. \& Gandini, M. R. Scientific use of the finite element method in Orthodontics. Dental Press J Orthod. 20, 119-125 (2015).

19. Korioth, T. W. \& Versluis, A. Modeling the mechanical behavior of the jaws and their related structures by finite element (FE) analysis. Crit Rev Oral Biol Med. 8, 90-104 (1997).

20. Goto, M., Yanagisawa, W., Kimura, H., Inou, N. \& Maki, K. A method for evaluation of the effects of attachments in aligner-type orthodontic appliance: Three-dimensional finite element analysis. Orthodontic Waves. 76, 207-214 (2019).

21. Savignano, R. et al. (2019) Biomechanical Effects of Different Auxiliary-Aligner Designs for the Extrusion of an Upper Central Incisor: A Finite Element Analysis. J Healthc Eng,,9687127.

22. Wang, C. Y. et al. Tension-compression viscoelastic behaviors of the periodontal ligament. J Formos Med Assoc. 111, 471-481 (2012).

23. Jiang, T., Wu, R. Y., Wang, J. K., Wang, H. H. \& Tang, G. H. Clear aligners for maxillary anterior en masse retraction: a 3D finite element study. Sci Rep. 10, 10156 (2020).

24. Meng, X. H. et al. Three-dimensional finite element analysis on en-mass retraction of anterior teeth with clear aligner. Zhonghua Kou Qiang Yi Xue Za Zhi. 54, 753-759 (2019).

25. Hennessy, J. \& Al-Awadhi, E. A. Clear aligners generations and orthodontic tooth movement. J Orthod. 43, 68-76 (2016). 
26. Sheng, Y., Guo, H. M., Bai, Y. X. \& Li, S. Dehiscence and fenestration in anterior teeth. (2020) Comparison before and after orthodontic treatment.J Orofac Orthop, 81,1-9.

27. Simon, M., Keilig, L., Schwarze, J., Jung, B. A. \& Bourauel, C. Treatment outcome and efficacy of an aligner technique-regarding incisor torque, premolar derotation and molar distalization. BMC Oral Health. 14, 68 (2014).

28. Castroflorio, T., Garino, F., Lazzaro, A. \& Debernardi, C. Upper-incisor root control with Invisalign appliances. J Clin Orthod. 47, 346-351 (2013).

29. Needham, R., Waring, D. T. \& Malik, O. H. Invisalign treatment of Class III malocclusion with lower-incisor extraction. J Clin Orthod. 49, 429-441 (2015).

30. Galan-Lopez, L., Barcia-Gonzalez, J. \& Plasencia, E. A systematic review of the accuracy and efficiency of dental movements with Invisalign®. Korean J Orthod. 49, 140-149 (2019).

31. Guan, X. et al. Clinical efficacy of clear aligners in treating bimaxillary protrusion. Zhonghua Kou Qiang Yi Xue Za Zhi. 52, 549-553 (2017).

32. Giancotti, A., Greco, M. \& Mampieri, G. Extraction treatment using Invisalign Technique. Prog Orthod. 7, 32-43 (2006).

33. Bertossi, D. et al. Histological evaluation of periodontal ligament in human after orthodontic treatment with piezosurgery and monolateral tooth dislocation and ligament distraction technique: a first morphologic and histologic evaluation. J Biol Regul Homeost Agents. 32, 9-13 (2018).

34. Kandil, S. (2016) The next big thing in clear aligner therapy: Enhancement of the digital workflow K Line Europe Germany Dentistry: 6:9.

35. Tawfik, A. H., El-Bialy, O. M. \& T. H Composite Polymers Transducers for Ultrasonic and Biological Applications. Ferroelectrics Letters Section. 30, 1-2 (2003).

36. Tarek El-Bialy, D. G. S. (2016) Orthodontic Biomechanics: Treatment of Complex Cases Using Clear Aligner, 1,1011.

\section{Figures}

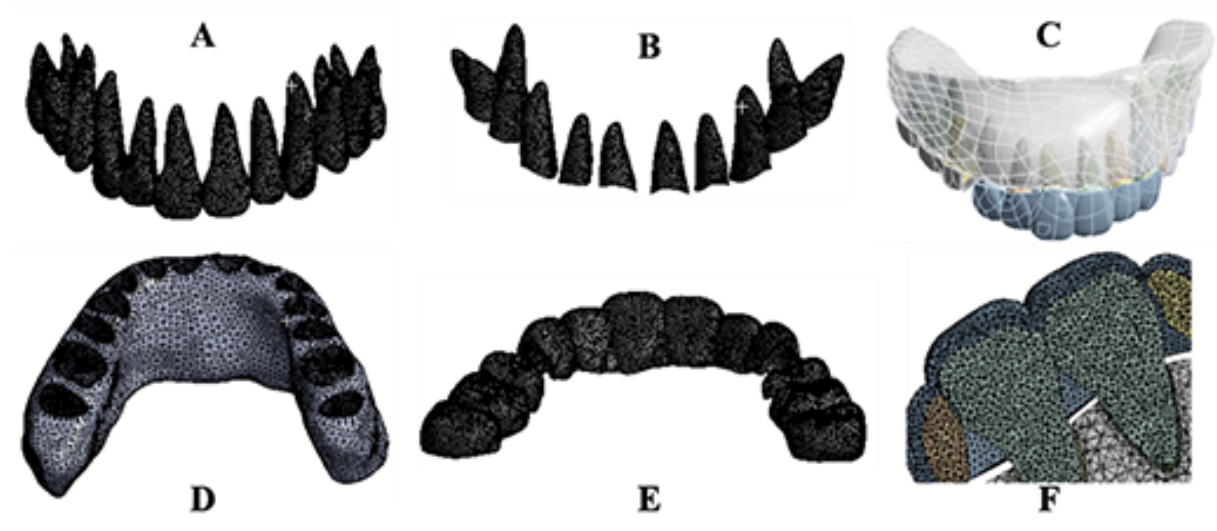

Figure 1

The three-dimensional finite element models of maxillary teeth, alveolar bone, PDL and CA. Maxillary teeth (A), Periodontal ligament (B), Assembly (C), Maxillary alveolar bone (D), Maxillary alveolar bone (D), Maxillary clear aligner (E), Assembly section (F). 
A

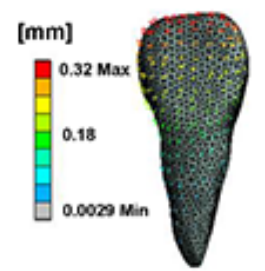

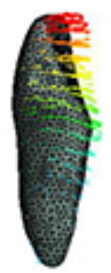

B

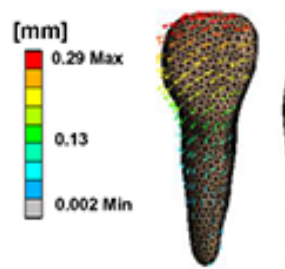

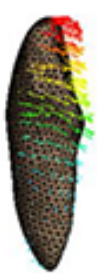

C

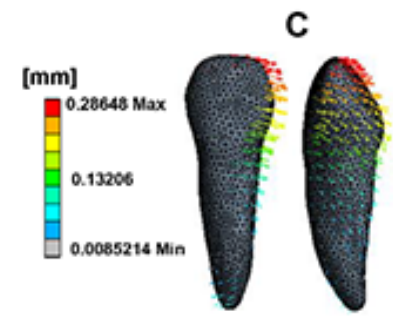

D

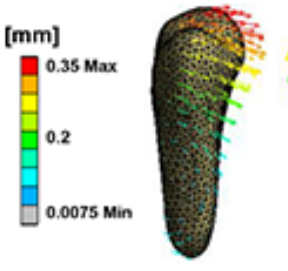

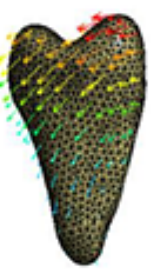
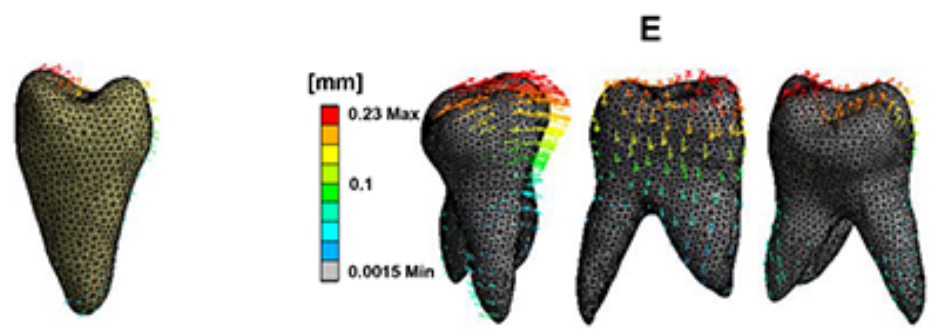

Figure 2

Cloud diagram of initial displacement trend of maxillary teeth. central incisor (A), lateral incisor, canine (B), second premolar (C), first molar (D). Front, middle, and back rows are palatal, mesial, and distal respectively. The direction and length of the arrow indicated the direction and size of displacement and deformation, respectively.

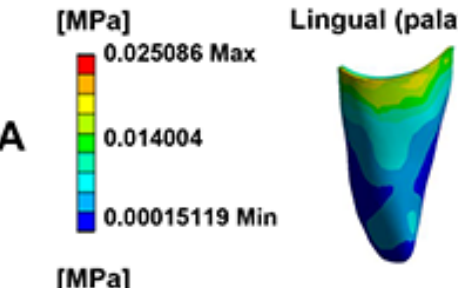

B

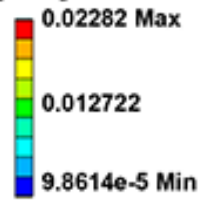

[MPa]

C $\underbrace{0.036898 \text { Max }}_{0.020711}$ [MPa]
$\mathrm{F}^{0.042274 \mathrm{Max}}$
D
0.023909
$0.00095172 \mathrm{Min}$
[MPa]

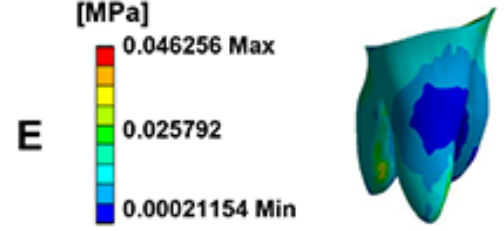

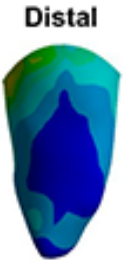
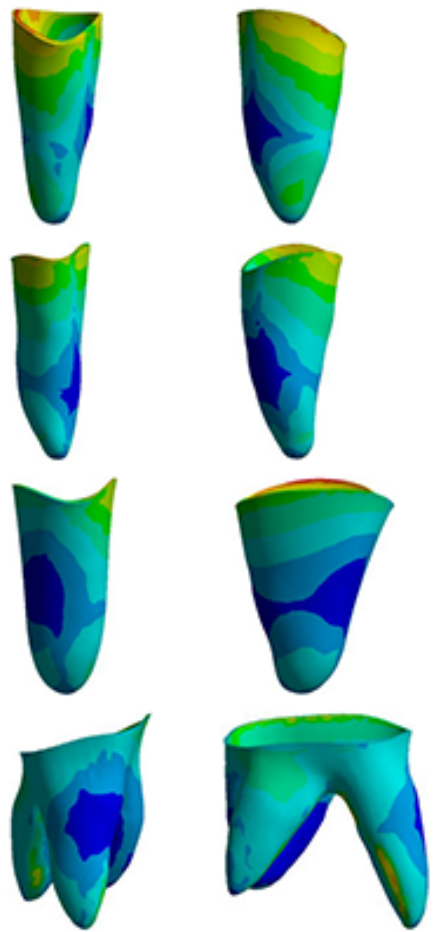
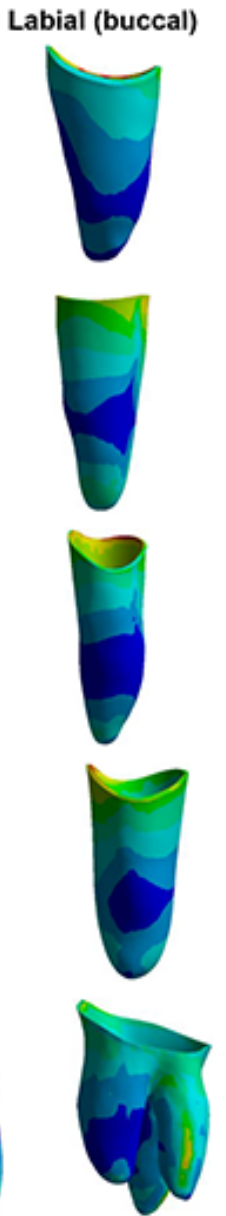
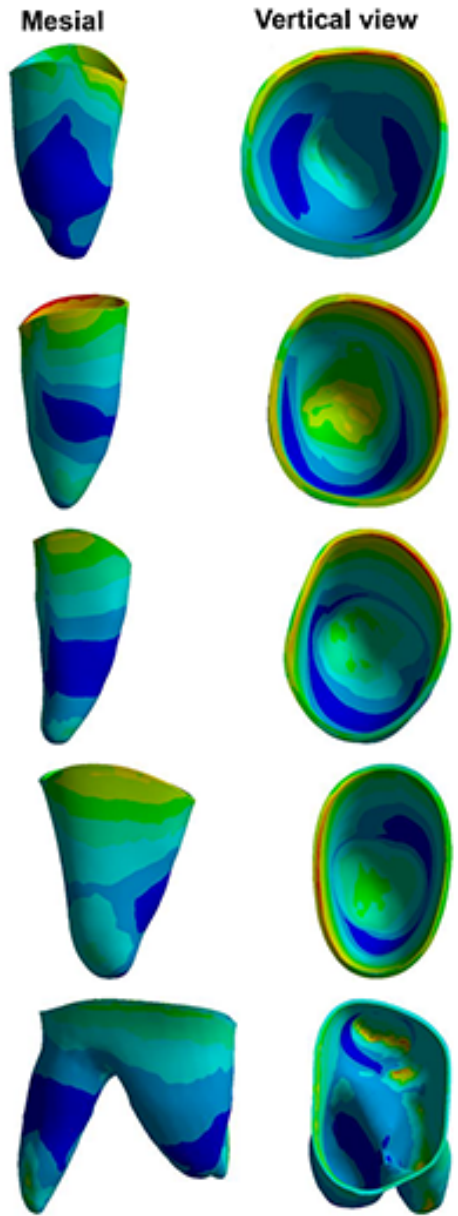

Figure 3 
Cloud diagram of the equivalent stress distribution of PDL in maxillary. Central incisor (A), lateral incisor (B), canine (C), second premolar (D), first molar(E).

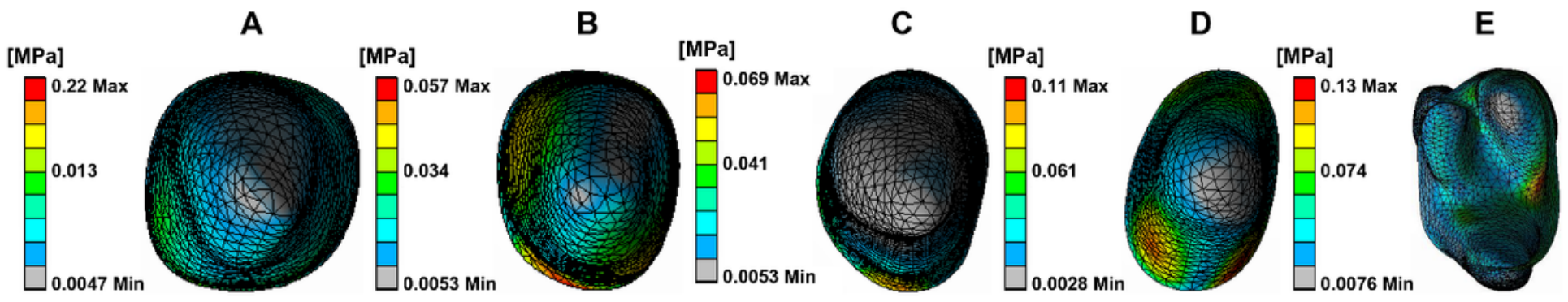

\section{Figure 4}

Cloud diagram of equivalent stress distribution of alveolar socket. Central incisor (A), lateral incisor (B), canine (C), second premolar (D), first molar (E).

A

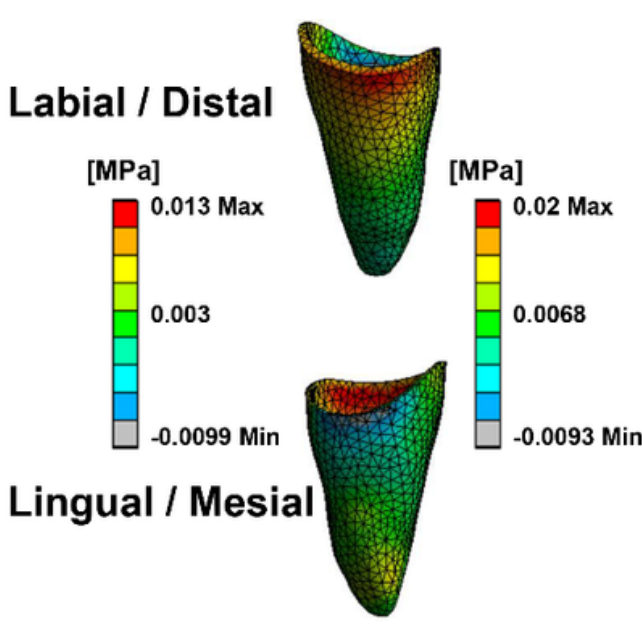

B

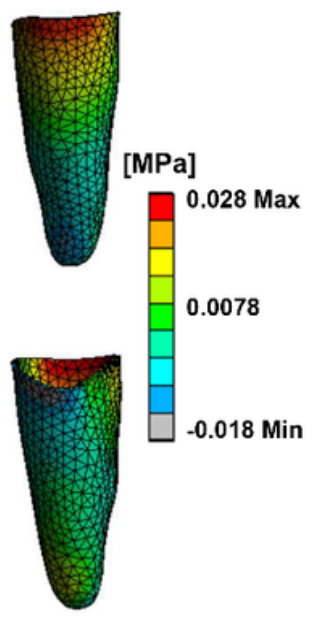

C

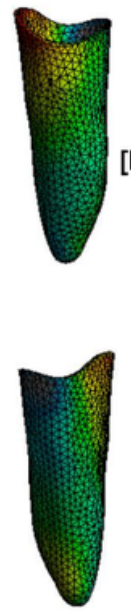

D
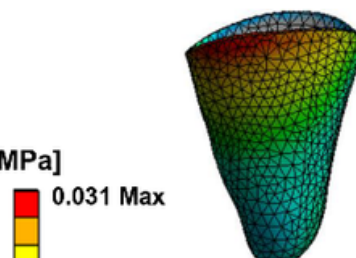

[MPa]

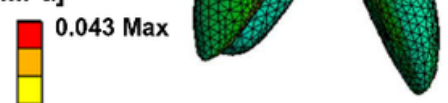

0.013

$-0.017 \mathrm{Min}$

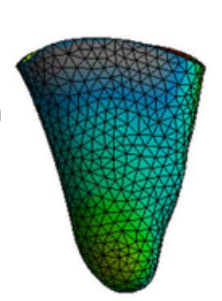

E

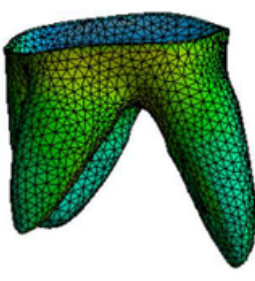

0.013

$-0.023 \mathrm{Min}$

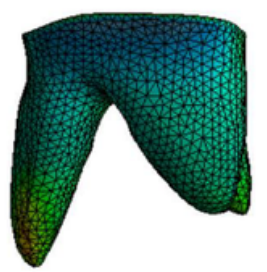

Figure 5

Cloud diagram of tensile and compressive stress of periodontal ligament in maxillary teeth. Central incisor (A), lateral incisor $(B)$, canine $(C)$, second premolar (D), first molar $(E)$. 
[mm]

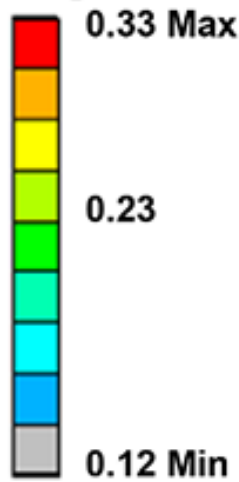

[mm]

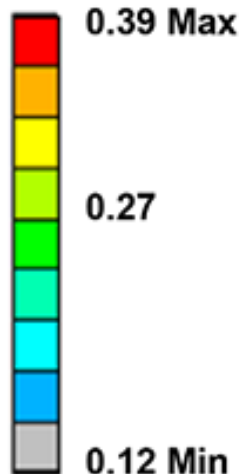

A

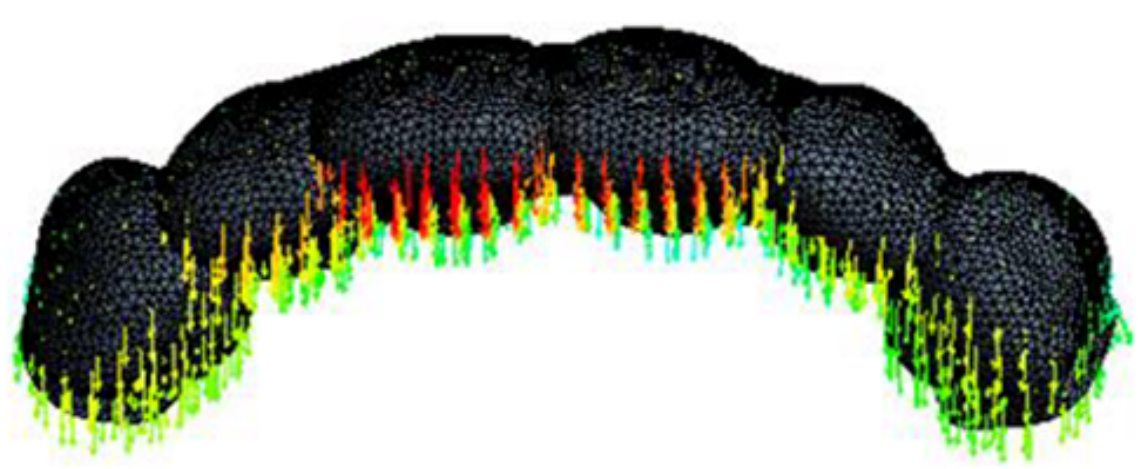

B

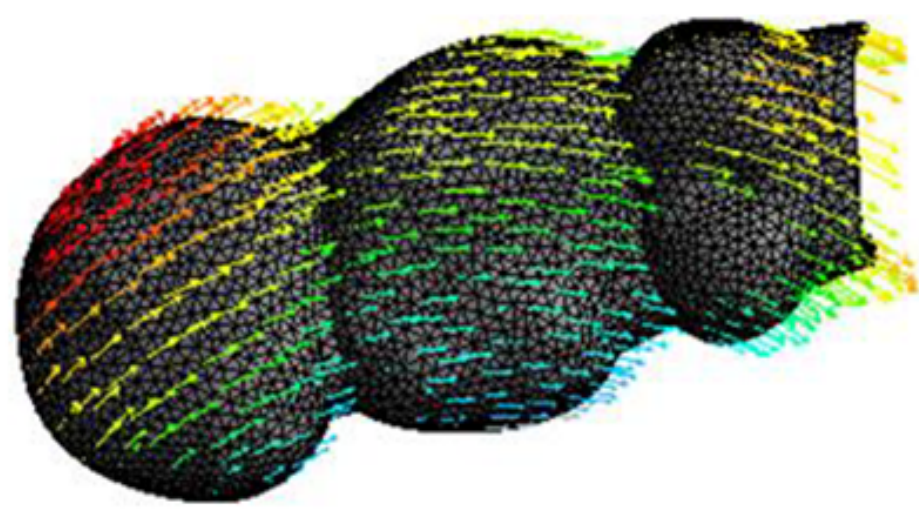

Figure 6

Cloud diagram of deformation trend of CA. Anterior area (A), Posterior area (B). The direction and length of the arrow indicate the direction and size of displacement and deformation, respectively. 

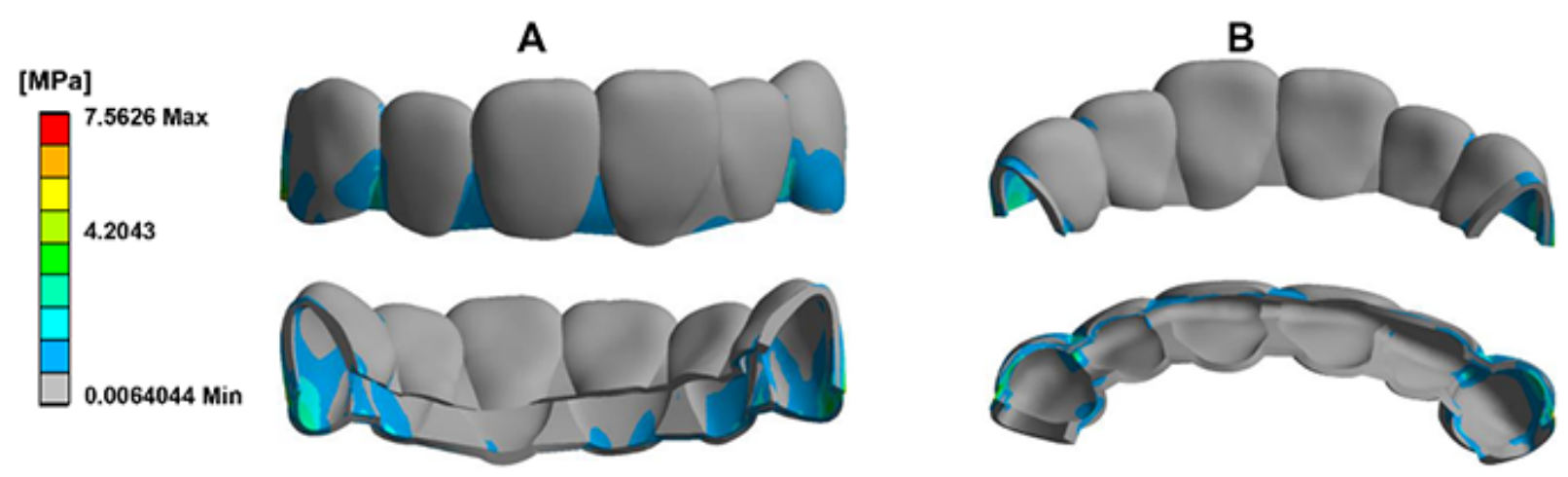

[MPa]
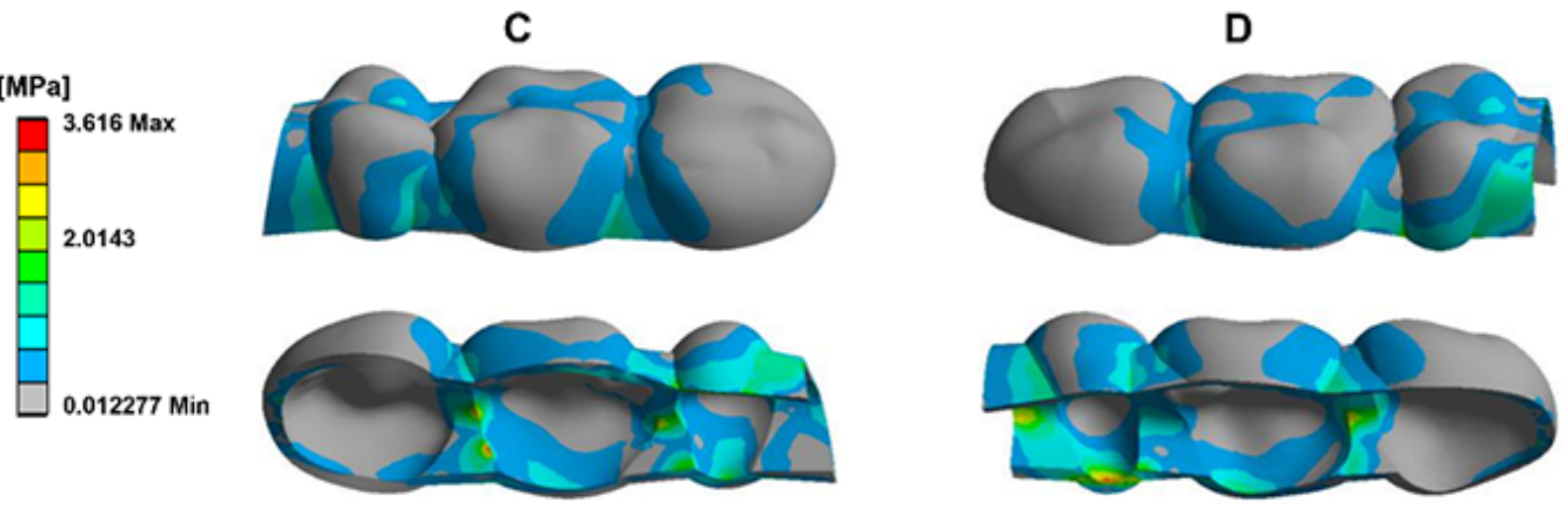

\section{Figure 7}

Cloud diagram of equivalent stress distribution of CA. Labiolingual side of anterior area ( $A$ and $B$ ), buccal and palatal sides of posterior area ( $C$ and $D)$. 